Research Article

\title{
Estimation of missing data in design of experiment and contingency table
}

\author{
D. S. Hooda' - M. S. Barak ${ }^{2}$ (1)
}

(C) Springer Nature Switzerland AG 2019

\begin{abstract}
Missing data in design of experiment and contingency table causes incomplete information which leads to more ambiguity in decision making process. Thus, to estimate missing data is an important and challenging problem. In the present paper estimation of missing data in design of experiment by applying the maximum entropy principle is described. An algorithm to estimate the missing values in a fuzzy matrix is defined and applied in estimation of missing data in contingency table.
\end{abstract}

Keywords Chi square statistics · Design of experiment · Maximum entropy principle · Fuzzy matrix · Fuzzy contingency table

\section{Introduction}

A set $A=\left\{a_{1}, a_{2}, \ldots, a_{m}\right\}$ of $m$ students each appears in set $B=\left\{b_{1}, b_{2}, \ldots, b_{n}\right\}$ of $m$ papers. The marks obtained by $m$ students in $n$ papers can be represented in a contingency table consisting a rectangular matrix of order $(m \times n)$ having $m n$ cells, where $m$ and $n$ are number of rows and columns representing characteristics of two attributes. In matrix notation contingency table is represented as given below (Table 1).

In the above table $O_{i j}$ is called the $(i, j)$ th cell which represents the frequency of characteristic of attributes $A$ and $\mathrm{B}$, where $r_{i}$ and $c_{j}$ are marginal row and column sum, $T$ is total sum respectively. The null hypothesis $H_{0}$ of independence of attributes against $H_{1}$ that attributes are dependent and can be tested by using $\mathrm{Chi}$ square test statistics i.e.

$\chi^{2}=\sum_{i=1}^{m} \sum_{j=1}^{n} \frac{\left(a_{i j}-e_{i j}\right)^{2}}{e_{i j}}$
In (1.1) $e_{i j}$ is the expected frequency corresponding to $(i, j)$ th cell having observed frequency $O_{i j}$ under the null hypothesis of independence and is given by:

$e_{i j}=\frac{r_{i} c_{j}}{T}$

where $T=\sum_{i=1}^{m} r_{i}=\sum_{j=1}^{n} c_{j}$.

A decision about $H_{0}$ is made by comparing the calculated value of Chi square from (1.1) with the tabulated value of Chi square for $(m-1)(n-1)$ degrees of freedom with $a \%$ level of significance. Hooda and Kumar [1] studied an information theoretic model to measure the dependence among the attributes in a contingency table by applying maximum entropy principle.

Zou and Xiao [2] proposed data analysis approach of soft sets to estimate missing data. Actually, this method is applied on weighted average of all possible choice values of the objects. The most interesting feature of this approach is that it can only compute the summation of all the parameters values individually. The summation values are used for decision making purpose, but the missing values remain unknown. Recently, Das et al. [3] has applied an algorithmic

M. S. Barak, ms_barak@igu.ac.in; D. S. Hooda, ds_hooda@rediffmail.com | 'G.J. University of Science and Technology Hisar, Hisar, India. ${ }^{2}$ Department of Mathematics, I.G. University Meerpur, Rewari, HR, India. 
Table 1 Contingency table

\begin{tabular}{llllllll}
\hline $\mathrm{A} / \mathrm{B}$ & $\mathrm{b}_{1}$ & $\mathrm{~b}_{2}$ & $\cdot$ & $\cdot$ & $\cdot$ & $\mathrm{b}_{\mathrm{n}}$ & \\
\hline $\mathrm{a}_{1}$ & $\mathrm{O}_{11}$ & $\mathrm{O}_{12}$ & $\cdot$ & $\cdot$ & $\cdot$ & $O_{1 n}$ & $r_{1}$ \\
$\mathrm{a}_{2}$ & $\mathrm{O}_{21}$ & $\mathrm{O}_{22}$ & $\cdot$ & $\cdot$ & $\cdot$ & $O_{2 n}$ & $r_{2}$ \\
$\cdot$ & $\cdot$ & $\cdot$ & $\cdot$ & $\cdot$ & $\cdot$ & $\cdot$ & $\cdot$ \\
$\cdot$ & $\cdot$ & $\cdot$ & $\cdot$ & $\cdot$ & $\cdot$ & $\cdot$ & $\cdot$ \\
$\cdot$ & $\cdot$ & $\cdot$ & $\cdot$ & $\cdot$ & $\cdot$ & $\cdot$ & $\cdot$ \\
$\mathrm{a}_{\mathrm{m}}$ & $\mathrm{O}_{m 1}$ & $\mathrm{O}_{m 2}$ & $\cdot$ & $\cdot$ & $\cdot$ & $O_{m n}$ & $r_{m}$ \\
& $c_{1}$ & $c_{2}$ & $\cdot$ & $\cdot$ & $\cdot$ & $c_{n}$ & $T$ \\
\hline
\end{tabular}

approach in estimation of the missing data in incomplete fuzzy soft set and interval valued fuzzy soft set which were introduced by Cagman and Enginoglu [4].

The rectangular matrix $H=\left[a_{i j}\right], i=1,2,3, \ldots, m$ and $j=1,2,3, \ldots, n$ is called fuzzy matrix if $a_{i j} \in[0,1]$ for every $i, j$. Similarly the contingency table can be called fuzzy contingency table if the entry $O_{i j} \in[0,1]$. It may be noted that every contingency table can be converted to fuzzy contingency table on dividing each entry by the largest entry or any convenient value near to the largest. If some values of data in the contingency table are found missing, then it is very essential to estimate these values in data analysis.

In the present communication, Yate's method and maximum entropy principle for estimation of mission data in design of experiment are described in Sect. 2. In Sect. 3, algorithm for estimation of missing data in a fuzzy matrix is explained and illustrated with an example and the same is applied in estimating the missing data in contingency table in Sect. 4. Conclusion and references are given in the end.

\section{Estimation of missing data in design of experiments}

The plots are designed in different blocks before administering treatments in the field of experiment. If one or more observations are missing due to natural calamities or by a pest or eaten by animals, it is cumbersome to estimate the missing value or values in the field trials as it is practically impossible to repeat the experiment under the similar conditions. Yates [5] suggested a method which was also described by Hooda [6] as given below:

"Substitute' $x$ ' for the missing value and after that selects that value of $x$ so as to minimize the error sum of squares".

Actually, the substituted value does not recover the best information; however, it gives the best estimate according to a criterion based on the least square method. For the randomized block experiment the missing yield is estimated as

$x=\frac{p P+q Q-T}{(p-1)(q-1)}$, where $p$ represents the number of treatments, $q$ is number of blocks, $P$ is the total of all plots receiving the same treatment as the missing plot, $Q$ represents total number of plots in the same block as the missing plot, $T$ is total of all plots. In the Latin square design, the formula corresponding to $(2.1)$ is

$x=\frac{p\left(P_{r}+P_{c}+P_{t}\right)-2 T}{(p-1)(q-1)}$

where $p$ represents the number of rows and columns of treatments, $P_{r}$ is total of row containing the missing plot, $P_{c}$ is the total of column containing the missing plot, $P_{c}$ represents the total of treatment contained in the missing plot, $T$ is the grand total.

In case more than one plot yields are missing, we substitute the average yield of available plots in all except one of these and substitute $x$ in this plot. We estimate ' $x$ ' by Yate's method and use this value to estimate the missing yields of other plots one by one.

Next we discuss the maximum entropy method due to Kapur and Keshvan [7]. If $x_{1}, x_{2}, \ldots, x_{n}$, are known yields and $x$ is the missing yield. We obtain the maximum entropy estimate for $x$ by maximizing:

$-\sum_{i=0}^{n} \frac{x_{i}}{T+x} \log \frac{x_{i}}{T+x}-\frac{x}{T+x} \log \frac{x}{T+x}$

Thus, we obtain

$\hat{x}=\left[x_{1}^{x_{1}} \cdot x_{2}^{x_{2}} \cdot x_{3}^{x_{3}} \ldots x_{n}^{x_{n}}\right]^{\frac{1}{T+y}}$

where $T=\sum_{i=1}^{n} x_{i}$, and the value given by (2.4) is called as maximum entropy mean of $x_{1}, x_{2}, x_{3}, \ldots, x_{n}$.

Similarly, if two values $x$ and $y$ are missing, $x$ and $y$ are determined from

$\hat{x}=\left[x_{1}^{x_{1}} \cdot x_{2}^{x_{2}} \cdot x_{3}^{x_{3}} \ldots x_{n}^{x_{n}}\right]^{\frac{1}{T+y}}$

$\hat{y}=\left[x_{1}^{x_{1}} \cdot x_{2}^{x_{2}} \cdot x_{3}^{x_{3}} \ldots x_{n}^{x_{n}}\right]^{\frac{1}{T+x}}$

The solution of (2.5) and (2.6) is given by 
$\hat{y}=\left[x_{1}^{x_{1}} \cdot x_{2}^{x_{2}} \cdot x_{3}^{x_{3}} \ldots x_{n}^{x_{n}}\right]^{\frac{1}{T}}$

Hence all the missing values have the same estimate and this does not change if the missing values are estimated one by one.

There are some drawbacks in the estimate (2.4) which are as given below:

1. $\hat{x}$ is rather unnatural. In fact $\hat{x}$ is always greater than the arithmetic mean of $x_{1}, x_{2}, x_{3}, \ldots, x_{n}$.

2. If two values are missing the maximum entropy estimated for each is the same as given by (2.7).

3. This is not very useful for estimating missing values in design of experiments.

The first drawback can be overcome by using generalized measure of entropy instead of Shannon entropy. If we use Burg's [8] measure given by $B(P)=\sum_{i=1}^{n} \log p_{i}$

Then we get the estimate $\hat{x}=\frac{x_{1}+x_{2}+\cdots+x_{n}}{n}=\bar{x}$. In fact we choose a value $\hat{x}$, which is as equal to $x_{1}, x_{2}, x_{3}, \ldots, x_{n}$ as possible and so we maximize a measure of equality. Since there are many measures of equality, therefore our estimate will also depend on the measure of equality we choose. The second drawback can be understood by considering the fact that the information theoretic estimate for a missing value depends on:

(a) The information available to us

(b) The purpose for which missing value is to be used.

The third drawback, as according to the principle of maximum entropy, we should use all the information given to us and avoid scrupulously using any information not given to us. But in design of experiments, we are given information about the structure of the design which we are not using this knowledge in estimating the missing values. Consequently, the estimate is not accurate; however, method defined in Sect. 2 can be applied to estimate the missing value $x_{i j}$ in contingency tables, where, the value $x_{i j}$ is to be chosen to minimize the measure of dependence $D$.

\section{An algorithm method for estimation of missing data in fuzzy matrix}

Let us consider a fuzzy matrix $X=\left[x_{i j}\right]_{m \times n}$ defined by Vasantha Kandasamy [9]. The basic concept of the fuzzy matrix is very simple and can be applied to many social and natural situations. Hooda and Jain [10] defined max-min and min-max binary operations on a set of fuzzy matrices and proved that it is a lattice under these operations.

Let $H=(U, E)=\left(h_{i j}\right)_{m \times n^{\prime}} i=1,2, \ldots, m$ and $j=1,2, \ldots, n$ be a fuzzy matrix having some missing values, where $U=\left\{d_{1}, d_{2}, \ldots, d_{m}\right\}$ be the set of values of an attribute and $E=\left\{e_{1}, e_{2}, \ldots, e_{n}\right\}$ be corresponding values of another attribute. Each missing value in $H$ is denoted by ${ }^{\prime * \prime}$ in the tabular representation. Now we explain an algorithm for estimating the missing value ${ }^{\prime * \prime}$ in $H$ which is analogous to algorithm due to Surjit et al. [7] for fuzzy soft matrix defined by Cagman and Enginoglu [4].

Step 1 Mean value $X_{j}$ for each attribute value $e_{j}, j=1,2, \ldots, n$ is computed as $X_{j}=\sum_{i=1}^{m} \frac{h_{i j}}{\left|u_{j}\right|}$, where $U_{j}=\left\{i \mid h_{i j} \neq *, 1 \leq i \leq m\right\}$.

Step 2 Average distance of each entry $h_{i j}, i=1,2, \ldots, m$ from the mean $X_{j}$ is denoted by $D_{a v g}\left(X_{j}\right)=\sum_{i=1}^{m} \frac{\left|X_{j}-h_{i j}\right|}{\left|U_{j}\right|} \forall j$, where $U_{j}=\left\{i \mid h_{i j} \neq *, 1 \leq i \leq m\right\}$.

Step 3 A pair of distance information $\left\{d X_{j}^{+}, d X_{j}^{-}\right\}$on either side of the mean $X_{j}$ is computed, where $d X_{j}^{+}=X_{j}+D_{a v g}\left(X_{j}\right)$ and $d X_{j}^{-}=X_{j}-D_{a v g}\left(X_{j}\right)$.

Step $4 h_{i j} j=1,2, \ldots, n$ for each $d_{i}, i=1,2, \ldots, m$ is compared with the mean $X_{j}, j=1,2, \ldots, n, h_{i j} \neq^{*}, 1 \leq i \leq m$. Let $n_{L}$ be the number of count for which $h_{i j}<X_{j}$ and $n_{G}$ be the number of count for which $h_{i j} \geq X_{j}$ for the corresponding attribute value $e_{j} j=1,2, \ldots, n$

Step 5 Utilizing $n_{L}$ and $n_{G}$, the probabilistic weights $w_{L}$ and $w_{G}$ of each object $d_{i}, i=1,2, \ldots, m$ are respectively defined as $w_{L_{i}}=n_{L} /\left(n_{L}+n_{G}\right)$ and $w_{G_{i}}=n_{G} /\left(n_{L}+n_{G}\right)$. Step 6 The unknown or missing entry in fuzzy matrix for attribute value $e_{j}$ is computed as $h_{i j}=\left(d X_{j}^{+} \times w_{G_{i}}\right)+\left(d X_{j}^{-} \times w_{L_{i}}\right), \quad$ w h e r e $h_{i j}=*, 1 \leq i \leq m, 1 \leq j \leq n$.

\section{Estimation of missing data in contingency table}

In this section we develop a method for estimation of missing data in incomplete contingency table by applying the algorithm proposed in Sect. 3. As mentioned earlier a contingency table can be transformed to fuzzy contingency table on dividing each entry by the largest entry or any convenient value near to the largest entry.

Let $X=\left(O_{i j}\right)_{m \times n}$ be the contingency table having missing data in some rows and columns with their corresponding marginal row and column sum totals are also missing in such a way that it is not possible to estimate any of these values by calculations. 
Step $1^{*}$ Consider incomplete matrix of rows and columns.

Step $2^{*}$ Divide each entry except the missing entries by the largest or any convenient value near to the largest.

Step $3^{*}$ Apply algorithm method proposed in Sect. 3.

\subsection{Numerical illustrations}

Let us consider the following incomplete contingency table for estimation of missing values (Table 2).

Step 1 Here we consider incomplete matrix only of rows and columns of the following contingency table (Table 3 ).

Step 2 We divide each entry by 25 and thus get the following incomplete matrix (Table 4).

Step 3 Mean of $b_{j}$ is denoted by $X_{j}, j=1,2, \ldots, 5$ is computed as given below:
Table 2 Incomplete contingency table

\begin{tabular}{lllllll}
\hline$A / B$ & $b_{1}$ & $b_{2}$ & $b_{3}$ & $b_{4}$ & $b_{5}$ & \\
\hline$a_{1}$ & 25 & 10 & 7 & 8 & 12 & 62 \\
$a_{2}$ & 9 & $*$ & 14 & $*$ & 10 & $*$ \\
$a_{3}$ & 6 & 18 & $*$ & 7 & $*$ & 51 \\
$a_{4}$ & 8 & 3 & $*$ & 19 & 23 & $*$ \\
& 48 & $*$ & 41 & $*$ & $*$ & 227
\end{tabular}

Step 6 Now, compare $O_{2 j}$ corresponding to $a_{2}$ with the corresponding mean $X_{j}, j=1,2,3,4,5$. As shown in algorithm Step 4 and Table 4 for $b_{1}, O_{21}\left\langle X_{1} ;\right.$ for $\left.b_{3}, O_{23}\right\rangle X_{3} ;$ for $h_{5}, h_{25}<X_{5}$. Hence, $n_{L}=2, n_{G}=1$.

Step 7 Probabilistic weights corresponding to the value $a_{2}$ of attribute $A$ are calculated as

$w_{L_{2}}=\frac{2}{3}, w_{G_{2}}=\frac{1}{3}$

$$
\begin{aligned}
& X_{1}=\frac{(1+0.36+0.24+0.32)}{4}=0.48, X_{2}=\frac{(0.4+0.72+0.12)}{3}=0.41, X_{3}=\frac{(0.28+0.56)}{2}=0.42 \\
& X_{4}=\frac{(0.32+0.28+0.76)}{3}=0.45, X_{5}=\frac{(0.48+0.4+0.92)}{3}=0.51
\end{aligned}
$$

Step 4 Find the average distance and average $\left(X_{j}\right)$, $\theta_{i j}, i=1,2,3,4$

$$
\begin{aligned}
& D_{\text {avg }}\left(X_{1}\right)=\frac{(|1-0.48|+|0.36-0.48|+|0.24-0.48|+|0.32-0.48|)}{4}=0.26 \\
& D_{\text {avg }}\left(X_{2}\right)=\frac{(|0.4-0.41|+|0.72-0.41|+|0.12-0.41|)}{3}=0.23 \\
& D_{\text {avg }}\left(X_{3}\right)=\frac{(|0.28-0.42|+|0.56-0.42|)}{2}=0.14 \\
& D_{\text {avg }}\left(X_{4}\right)=\frac{(|0.32-0.45|+|0.28-0.45|+|0.76-0.45|)}{3}=0.21 \\
& D_{\text {avg }}\left(X_{1}\right)=\frac{(|0.48-0.51|+|0.4-0.51|+|0.92-0.51|)}{3}=0.21
\end{aligned}
$$

Step 5 Distance pair $\left\{d X_{1}^{+}, d X_{1}^{-}\right\}$for the mean $X_{i}$ is calculated as:

$d X_{1}^{+}=(0.48+0.26)=0.74, d X_{1}^{-}=(0.48-0.26)=0.22$

Hence, $\left(d X_{1}^{+}, d X_{1}^{-}\right)=(0.74,0.22)$ similarly, other distance pairs are computed as
Step 8 Now, the unknown entry $\mathrm{O}_{22}$ in Table 4 is computed as

$$
\begin{aligned}
O_{22} & =\left[\left(d X_{2}^{+} \times w_{G_{2}}\right)+\left(d X_{2}^{-} \times w_{L_{2}}\right)\right] \\
D_{1} & =\left[0.64 \times \frac{1}{3}+0.18 \times \frac{2}{3}\right] \\
& =0.21+0.12=0.33 .
\end{aligned}
$$

$\left(d X_{2}^{+}, d X_{2}^{-}\right)=(0.64,0.18),\left(d X_{3}^{+}, d X_{3}^{-}\right)=(0.56,0.28),\left(d X_{4}^{+}, d X_{4}^{-}\right)=(0.66,0.24)$

$\left(d X_{5}^{+}, d X_{5}^{-}\right)=(0.72,0.3)$. 
Table 3 Incomplete matrix of contingency table

\begin{tabular}{llllll}
\hline $\mathrm{A} / \mathrm{B}$ & $\mathrm{b}_{1}$ & $\mathrm{~b}_{2}$ & $\mathrm{~b}_{3}$ & $\mathrm{~b}_{4}$ & $\mathrm{~b}_{5}$ \\
\hline $\mathrm{a}_{1}$ & 25 & 10 & 7 & 8 & 12 \\
$\mathrm{a}_{2}$ & 9 & $*$ & 14 & $*$ & 10 \\
$\mathrm{a}_{3}$ & 6 & 18 & $*$ & 7 & $*$ \\
$\mathrm{a}_{4}$ & 8 & 3 & $*$ & 19 & 23 \\
\hline
\end{tabular}

Table 4 Incomplete fuzzy matrix

\begin{tabular}{llllll}
\hline $\mathrm{A} / \mathrm{B}$ & $\mathrm{b}_{1}$ & $\mathrm{~b}_{2}$ & $\mathrm{~b}_{3}$ & $\mathrm{~b}_{4}$ & $\mathrm{~b}_{5}$ \\
\hline $\mathrm{a}_{1}$ & 1 & 0.4 & 0.28 & 0.32 & 0.48 \\
$\mathrm{a}_{2}$ & 0.36 & $*$ & 0.56 & $*$ & 0.4 \\
$\mathrm{a}_{3}$ & 0.24 & 0.72 & $*$ & 0.28 & $*$ \\
$\mathrm{a}_{4}$ & 0.32 & 0.12 & $*$ & 0.76 & 0.92 \\
\hline
\end{tabular}

Table 5 Complete contingency table

\begin{tabular}{lllllll}
\hline $\mathrm{A} / \mathrm{B}$ & $\mathrm{b}_{1}$ & $\mathrm{~b}_{2}$ & $\mathrm{~b}_{3}$ & $\mathrm{~b}_{4}$ & $\mathrm{~b}_{5}$ & \\
\hline $\mathrm{a}_{1}$ & 25 & 10 & 7 & 8 & 12 & 62 \\
$\mathrm{a}_{2}$ & 9 & 8 & 14 & 10 & 10 & 51 \\
$\mathrm{a}_{3}$ & 6 & 18 & 10 & 7 & 11 & 51 \\
$\mathrm{a}_{4}$ & 8 & 3 & 11 & 19 & 23 & 64 \\
& 48 & 39 & 41 & 44 & 56 & 227 \\
\hline
\end{tabular}

Similarly, other missing values are calculated as given below:

$O_{24}=0.38, O_{33}=0.38, O_{35}=0.44, O_{43}=0.42$

Step 9 Next multiplying each one by 25 , we get the missing data by approximating to the integral value of the Table 2 up to two decimals as given below:

$O_{22}=8, O_{24}=10, O_{33}=10, O_{35}=11, O_{43}=11$.

Step 10 Finally, putting these values in Table 2, we obtain the following complete contingency table (Table 5).

\section{Discussion and conclusion}

The estimation of the missing values is very important area in many fields of research like agriculture, economics, and science laboratory and data management. When observed valued are found missing due to nature climates or any other regions and are very difficult to repeat the experiments for example the field trials of agriculture crops or disrupted due to nature climates, can the technique to predict or estimate these value are need to be developed. Various scientist and technocrats have suggested different method valuably the max entropy method described by Kapur [7] and that have play an better role then Yate
[5] methods for estimating the missing data. Contingency tables have applications in economics, agriculture, and laboratory and data management to study independence of attributes. Kullback [11] discussed contingency tables from MDI principle point of view. Ku and Kullback [12] used a similar approach to discuss interactions in multidimensional contingency tables.

In the present paper a novel method of estimating missing data in contingency table has been evolved by applying algorithm for estimating missing values in a fuzzy matrix and that has been illustrated with numerical example. It is hoped that this technique would find applications in economics, laboratory, data analysis and data management.

\section{Compliance with ethical standards}

Conflict of interest On the behalf corresponding author states that there is no conflict of interest.

\section{References}

1. Hooda DS, Kumar P (2013) Information theoretic method for dependence analysis and missing data estimation. Am J Theor Appl Stat. https://doi.org/10.11648/j.ajtas.20130202.12

2. Zou Y, Xias Z (2008) Data analysis approaches of soft sets under incomplete information. Knowl Based Syst 21:941-945

3. Das $S$ et al (2017) An algorithm approach for predicting unknown information in incomplete fuzzy soft set. Arab J Sci Eng. https://doi.org/10.1007/s13369-017-2591-2

4. Cagman N, Enginogu S (2012) Fuzzy soft set theory and its applications in decision making. Iran J Fuzzy Syst 9(1):109-119

5. Yates F (1933) The analysis of replicated experiments when the field experiments are incomplete. Emp J Exp Agric 1:129-142

6. Hooda DS (2011) Maximum entropy method for estimation of missing data, International encyclopedia of statistical science. Springer, Berlin. https://doi.org/10.1007/978-3-642-04898 $-2 \_622$

7. Kapur JN, Keshvan HK (1992) Entropy optimization principles with applications. Academic Press, San Diego

8. Burg JP (1970) The relationship between maximum entropy spectra and maximum likelihood. In: Childers DG (ed) Modern spectra analysis. pp 130-131

9. Vasantha Kandasamy WB et al (2007) Elementary fuzzy matrix theory and fuzzy models for social scientists. Automation, Los Angles

10. Hooda DS, Jain D (2012) Measures of information on fuzzy matrix and fuzzy binary relations. J Comb Inf Syst Sci 37:1-18

11. Kullback S (1959) Information theory and Statistics. Wiley, New York

12. Ku HH, Kullback S (1968) Interactions in multidimensional contingency tables. J Res Nat B Sci 72-B:8159

Publisher's Note Springer Nature remains neutral with regard to jurisdictional claims in published maps and institutional affiliations. 